\title{
Die sogenannten Kochschen Postulate
}

Von Alfred Grafe

\section{Einleitung}

Zu den großen medizinhistorischen Gedenktagen zählt auch der 24. März 1882, an dem Robert Koch in einem Vortrag und mit Labor-Demonstrationen am Beispiel der Tuberkulose als erster unwiderlegbar bewiesen hatte, daß Mikroben nicht Folge, sondern Ursache von Infektionskrankheiten sind.

Die gleichen experimentellen Ziele hatte er 1876 beim Milzbrand und 1878 bei Wundinfektionskrankheiten, doch der gleiche Erfolg wie bei der Tbc blieb ihm versagt, weil die Labortechniken und die Laborgeräte für einen schlüssigen Beweis nicht ausgereicht hatten.

In den drei entsprechenden Publikationen hat Robert Koch sowohl seine nicht ausreichenden Beweisführungen bei Milzbrand und bei Wundinfektionen als auch seine unwiderlegbar-schlüssigen bei der Tuberkulose dargelegt. Die letzteren lauten ${ }^{1}$ : Um zu beweisen, daß die Tuberkulose eine durch die Einwanderung der Bazillen veranlaßte und in erster Linie durch das Wachstum und die Vermehrung derselben bedingte parasitische Krankheit sei, mußten die Bazillen vom Körper isoliert, in Reinkulturen solange fortgezüchtet werden, bis sie von jedem etwa noch anhängenden, dem tierischen Organismus entstammenden Krankheitsprodukt befreit sind, und schließlich durch die Übertragung der isolierten Bazillen auf Tiere dasselbe Krankheitsbild der Tuberkulose erzeugt werden, welches erfahrungsgemä $\beta$ durch Impfung mit natürlich entstandenen Tuberkelstoffen erhalten wird.

Da mit dem von allen anerkannten Tuberkulose-Beweis die Ätiologische Ära der Infektionskrankheiten begann, wurden und werden besonders die von Koch dargelegten drei Tbc-Beweisschritte als Muster des experimentellen Vorgehens bei der Aufklärung der Ursache einer Infektionskrankheit betrachtet.

In «Die Methoden der Bakterienforschung» ${ }^{2}$ von Ferdinand Hueppe, einem der ersten Mitarbeiter von Robert Koch, finden wir für einige auch von Koch als wesentlich herausgestellte Gedankengänge die Anwendung des Wortes «Postulat» im Sinne einer Aufforderung, sie bei Experimenten zur Ätiologie einer Infektionskrankheit zu beachten. Das ist z. B. die Frage der 
Verwendung von Tieren, die für die betreffende Krankheit nachweislich empfänglich sind ${ }^{3}$; die Verwendung eines einzelnen Keimes als Ausgangspunkt für eine Reinkultur ${ }^{4,5}$ sowie das Problem des festen und des durchsichtigen Nährbodens ${ }^{6}$, um Koch das Primat des Nähragars zu sichern.

Einer seiner beiden ersten Mitarbeiter im kaiserlichen Gesundheitsamt, Friedrich Löffler, würdigte Koch zu dessen 60. Geburtstag in der Deutschen Medizinischen Wochenschrift. Auch er verwendet das «Postulat» im obengenannten Sinn: Die Auffindung der Erreger war zunächst die Hauptaufgabe. Für den Beweis, daß ein Mikroorganismus als Erreger einer bestimmten Krankheit anzusehen ist, stellte Koch drei Postulate auf: konstanter Nachweis des betreffenden Organismus in allen Fällen der Krankheit, Gewinnung des Organismus in Reinkultur, frei von jeder Spur von körperlichen Bestandteilen des erkrankten Individuums, und Wiedererzeugung der Krankheit mittels zuverlässiger Reinkulturen. In seiner ausgezeichneten Arbeit über die Ätiologie der Wundinfektionskrankheiten hat er diese seine Prinzipien scharf präzisiert. Dieselbe ist daher das Muster und Vorbild geworden für alle späteren Arbeiten auf ätiologischem Gebiet.

Löffler bezieht sich auf die Wundinfektions-Arbeit und stellt sie im Gegensatz zu Koch als die bedeutendste für die ätiologische Forschung heraus.

Beide Arbeiten, die über die Wundinfektionen von 1878 und die Tuberkulose-Arbeit von 1882 oder die von 1884, werden später zitiert, wenn im Zusammenhang mit ätiologischer Forschung das Wort «Postulat» verwendet wird. Dessen Sinn erfährt dabei einen wesentlichen Wandel: Aus Hueppes Aufforderung zur Beachtung bestimmter Kochscher experimenteller Schritte und aus Löfflers Kochschen Prinzipien werden unabdingbare Forderungen, die Koch selbst aufgestellt haben soll.

Schadewaldt geht in seiner Gedenkrede zum 100. Jahrestag der Entdekkung des Tuberkulose-Erregers noch darüber hinaus, indem er schreibt: ... deren Postulate der frühere Lehrer Kochs Jakob Henle in seinem klassischen Werk über «Miasmen und Kontagien» von 1840 programmatisch umschrieben hatte. Darin erwähnt er bereits die später nach ihm benannten Postulate, die freilich erst Koch insbesondere bei der Entdeckung des Tuberkelbazillus in die Realität umsetzen konnte und die entscheidend mit dazu beitrugen, daß Kochs Arbeiten sofort von der wissenschaftlichen Öffentlichkeit anerkannt wurden. Sie bestanden aus den vier Forderungen ...

Nun gibt es also vier Kochsche Forderungen in der Tuberkulose-Arbeit aus dem Jahre 1882, und aus den Kochschen wurden Henle-Kochsche 
Postulate. So finden wir nicht nur im deutsch-, sondern auch im englischsprachigen Schrifttum eine Fülle von Meinungen über Kochs Postulate als Grundforderungen für das ätiologische Experiment ${ }^{7}$. Ausgangspunkt sowohl für englische wie für deutsche Arbeiten könnte eine Publikation von Thomas Rivers aus dem Jahre 1937 sein, wo die Postulate als strenge Forderungen Kochs gedeutet worden sind.

Da die Basis der von den Autoren hergeleiteten Postulate verschiedene Arbeiten Kochs sind, können sie auch nicht übereinstimmen.

So sind zwei Fragen zu klären: Gibt es Kochsche Postulate im Sinne unabdingbarer Forderungen, und gibt es einen Hinweis für die Annahme Henle-Kochscher Postulate?

\section{Gibt es Henle-Kochsche Postulate?}

Man entwertet die ausgezeichnete deduktive Literaturarbeit Henles nicht, wenn man feststellt, daß sie in seiner Zeit wenig Beachtung fand und für Koch aus vielerlei Gründen keine Bedeutung gehabt hat. Man kann sich ersparen, diese Gründe aus der Arbeit selbst herzuleiten, da wir einfachere und leichter erreichbare haben.

Als erster wäre Robert Koch selbst zu zitieren. In seiner 1909 vor der Akademie der Wissenschaften in Berlin gehaltenen Antrittsrede finden wir: ... so möchte ich zunächst erwähnen, daß ich auf der Universität keine unmittelbare Anregung für meine spätere wissenschaftiche Richtung empfangen habe ... Dennoch möchte ich einiger meiner damaligen Lehrer in Dankbarkeit gedenken, nämlich des Anatomen Henle...

Weiterhin gibt es zwei namhafte Zeugen dafür, daß sich Jakob Henle später nicht, auch nicht als Anatom in Göttingen, mit Fragen der Ursache von Infektionskrankheiten befaßt hat.

Die Einleitung im Nachdruck der Arbeit Henles in der von Karl Sudhoff herausgegebenen Reihe «Klassiker der Medizin» schrieb der in Leipzig tätige Pathologe und Anatom Felix Marchand. Wir lesen: Um so überraschender mag es manchem ferner Stehenden sein, daß wir als klassische Leistung hier eine Jugendarbeit des kaum 30jährigen Mannes bringen, die seinem eigentlichen späteren Lebenswerk ferner liegt ...

Ein weiterer Zeuge ist Eli Metschnikoff, der nach Göttingen kam, als Koch diese Stadt verließ. Er schrieb ${ }^{8}:$ Als ich 1866 bei Henle in Göttingen in einer Zeit arbeitete, als es ernsthafte Untersuchungen über mikroskopische 
Agentien infektiöser Krankheiten gab, blieb er indifferent und ... zu keiner Zeit kam in seinem Laboratorium die Frage ansteckender Krankheiten auf.

Somit ist die Frage nach der Existenz von Henle-Kochschen Postulaten zu verneinen.

\section{Was sind Kochsche Postulate?}

Da in den Arbeiten von Koch, die einen Bezug zur Ätiologie von Infektionskrankheiten haben, das Wort Postulat nicht zu finden ist, kann es auch keine authentische Übernahme der Kochschen Postulate durch andere geben. Man kann nur versuchen herauszufinden, ob es von Koch aufgestellte Grundsätze gibt, die etwas Immergültiges für die ätiologische Forschung aussagen. Sie sollten in unserer Zeit ebenso anwendbar sein wie vor 100 Jahren, ohne aber den Anspruch zu erheben, so alleingültig zu sein, wie es in Büchern und Publikationen den Anschein hat, wenn die Kochschen Postulate behandelt werden. Beginnen wir mit 1882.

Der erfolgreiche und von der wissenschaftlichen Welt voll anerkannte Abschluß der Tbc-Untersuchungen ließ nicht nur Koch erhoffen, daß mit den bei der Tuberkulose angewendeten experimentellen Methoden das Verfahren zur Aufklärung der Ätiologie von bakteriell bedingten Infektionskrankheiten gefunden sei, zumal auch in anderen Instituten nach den Kochschen Prinzipien mit Erfolg gearbeitet wurde.

Koch mußte seine Meinung revidieren, als es ihm und seinen Mitarbeitern bei der Cholera nicht gelungen war, ein geeignetes Versuchstier zu finden, in dem mit Reinkulturen die Infektion induziert werden konnte. Seine Gegner in der Cholera-Kommission, besonders der Antikontagionist Virchow und der Lokalist Pettenkofer, verwendeten diesen Mangel der fehlenden Tierversuche als Argument gegen die Auffassung der Cholera-Arbeitsgruppe um Koch, sie hätten den Erreger der Cholera gefunden.

Kochs Gegenargumente sind im Bericht der zweiten Konferenz zur Erörterung der Cholerafrage 1885 nachzulesen ${ }^{9}$. Er verwies auf die gleichgelagerten Fälle bei der Rekurrens-Spirochaete als dem Erreger des Rückfallfiebers sowie auf das Mycobakterium leprae und hält mit folgenden Argumenten für bewiesen, daß sein Bakterium der Erreger der Cholera ist:

1 Die Cholera-Bakterien sind in allen Fällen von Cholera vorgekommen, und 
2 Die Cholera-Bakterien sind bei keiner anderen Krankheit gefunden worden.

Mit dieser Argumentation unterscheidet Koch zwei Phasen seiner ätiologischen Arbeiten. Die erste umfaßt die Arbeiten vom Milzbrand bis zur Tuberkulose mit dem Ziel nachzuweisen, daß Bakterien nicht eine Folge, sondern die Ursache einer Infektionskrankheit sind. Als am Beispiel der Tuberkulose dieser Nachweis glänzend gelungen war, da begann die zweite, die ätiologische Phase, in der es die Frage nach Folge oder Ursache nicht mehr gab.

Die Kochsche Darstellung der Entwicklung zur Ätiologischen Ära finden wir in seinem Vortrag «Über bakteriologische Forschung» aus dem Jahre 1890, publiziert 1891: Der Gedanke, daß Mikroorganismen die Ursache der Infektionskrankheiten sein müßten, ist zwar von einzelnen hervorragenden Geistern schon sehr frühzeitig ausgesprochen, aber die allgemeine Meinung konnte sich damit nicht recht vertraut machen und verhielt sich gegenüber den ersten Entdeckungen auf diesem Gebiet sehr skeptisch. Um so mehr war es geboten, gerade in den ersten Fällen mit unwiderleglichen Gründen den Beweis zu führen, daß die bei einer Infektionskrankheit aufgefundenen Mikroorganismen auch wirklich die Ursache dieser Krankheit seien. Damals war der Einwand immer noch berechtigt, daß es sich um ein zufälliges Zusammentreffen von Krankheit und Mikroorganismen handeln könne, das letztere also nicht die Rolle von gefährlichen Parasiten, sondern von harmlosen Schmarotzern spielten, welche erst in den erkrankten Organen die im gesunden Körper fehlenden Existenzbedingungen fänden ...

Wenn es sich nun aber nachweisen ließ: erstens, daß der unter Verhältnissen, welche den pathologischen Veränderungen und dem klinischen Verlauf der Krankheit entsprechen; zweitens, daß er bei keiner anderen Krankheit als zufälliger und nicht pathogener Schmarotzer vorkommt; und drittens, da $\beta$ er, von dem Körper vollkommen isoliert und in Reinkulturen hinreichend oft umgezüchtet, imstande ist, von neuem die Krankheit zu erzeugen; dann konnte er nicht mehr zufälliges Akzidens der Krankheit sein, sondern es lie $\beta$ sich in diesem Falle kein anderes Verhältnis mehr zwischen Parasit und Krankheit denken, als daß der Parasit die Ursache der Krankheit ist.

Dieser Beweis hat sich denn nun auch in vollem Umfange für eine Anzahl von Infektionskrankheiten führen lassen, so für ... Dabei hat sich nun aber weiter ergeben, daß auch in allen den Fällen, in welchen es gelungen ist, bei einer Infektionskrankheit das regelmäßige und ausschließliche Vorkommen von 
Bakterien nachzuweisen, letztere sich niemals wie zufällige Schmarotzer, sondern wie die bereits sicher als pathogen erkannten Bakterien verhielten. Wir sind deshalb wohl jetzt schon zu der Behauptung berechtigt, daß, wenn auch nur die beiden ersten Forderungen der Beweisführung erfüllt sind, wenn also das regelmäßige und ausschließliche Vorkommen des Parasiten nachgewiesen wurde, damit der ursächliche Zusammenhang zwischen Parasit und Krankheit auch vollgültig bewiesen ist.

Somit hielt Koch 1890 die 1882 bei der Tuberkulose aufgestellten Beweisschritte nicht mehr für allein maßgebend, sondern er ergänzte sie um gleichwertige andere,

- das regelmäßige Vorkommen und

- das ausschließliche Vorkommen eines spezifischen Keimes bei einer Infektionskrankheit.

Diese zwei Anforderungen schließen den Tierversuch nicht mehr notwendigerweise ein, sie erfordern aber nach wie vor das Experiment mit Reinkulturen.

Aus dieser Kochschen Darstellung des experimentellen Weges zur Ätiologie einer Infektionskrankheit ergibt sich, daß man zwar von Kochschen Postulaten als Prinzipien sprechen kann, aber nicht von den, heute noch alleingültigen Postulaten im Sinne von unabdingbaren Forderungen, die andere Wege der Beweisführung als zweitklassig oder sogar als nichtbeweisend abwerten.

Diese Kochschen Postulate stehen nicht in seinen experimentellen Arbeiten vom Milzbrand bis zur Tuberkulose, sondern sie sind im Bericht der Zweiten Konferenz zur Erörterung der Cholerafrage vom Mai 1885 angedeutet und in seinem 1891 publizierten Berliner Vortrag «Über bakteriologische Forschung» eingehend dargelegt.

\section{Anmerkungen}

1 Gesammelte Werke von Robert Koch, Verlag Georg Thieme, Leipzig 1912, S.5-25; $61-108 ; 428-445$.

2 F. Hueppe, Die Methoden der Bakterien-Forschung, 4. Aufl., Kreidel's Verlag, Wiesbaden 1889.

3 In Hueppe: S. 359.

4 In Hueppe: S. 168.

5 In Hueppe: S. 284. 
6 In Hueppe: S. 285.

7 Zinsser/Bayne-Jones, A textbook of Bacteriology, 8. Lil. 1928, S.12/13.

Th. Rivers, Viruses and Koch's Postulates, J. Bact. 33, 1937; 1-12.

Topley and Wilson's, Principles of Bacteriology and Immunity, 4. Ed. 1955, S. 1140.

Microbiology, 4.Ed., Harper \& Row, Publ. 1968, S. 10.

R. Y.Stanier et al., General Microbiology, 3. Ed. 1971, S. 16.

F. M. Burnet, Principles of Animal Virology, 2. Ed. 1960, S.2.

S. E. Luria, General Virology, 1953, S. 32 .

H. A. Lechevalier and M. Solotorovsky, Three Centuries of Microbiology, 1965, S. 65.

A. P. Waterson and L. Wilkinson, An Introduction to the History of Virology, 1978, S. 67.

Medizinische Mikrobiologie, Springer-Verlag, 2. Aufl. 1968, S. 189.

Lehrbuch der Medizinischen Mikrobiologie, 3. Auflage, G. Fischer Verlag, Stuttgart, 1968, S.3.

8 In: Three Centuries of Microbiology, 1965, S. 65.

9 Gesammelte Werke von Robert Koch, 1912, 2. Band, Erster Teil, S. 89.

\section{Literatur}

J. Henle, Von den Miasmen und Kontagien und von den miasmatisch-kontagiösen Krankheiten. Klassiker der Medizin, Verlag J.A.Barth, Leipzig, 1910.

F. Loeffler, Robert Koch. Zum 60. Geburtstage. Cbl. Bakt. I./Orig. 35 401-15, 1904.

F.Loeffler, Zum 25jährigen Gedenktage der Entdeckung des Tuberkelbacillus, Deutsche Med. W schr. 33, 449-51, 489-95.

Th. Rivers, Viruses and Koch's Postulates, J. Bact. 33, 1-12, 1937.

H. Schadewaldt, Zur Geschichte der Entdeckung des Tuberkelbakteriums, Bundesgesundhbl. $25,121-33 ; 1982$.

J.Schwalbe, Gesammelte Werke von Robert Koch, Bd.1 und 2. Verlag Georg Thieme, Leipzig, 1912. 


\section{Summary}

It is shown that there exists neither Kochs' nor Henle-Kochs' postulates in the sense of permanently and exclusively valid demands, presumedly defined by Koch as cited in most microbiological or virological books and in corresponding publications.

Robert Koch did not set up dictums but in different papers he described the principles for this experiments to prove his statements conclusively.

Up to 1882 Koch's only aim was to prove that bacteria are not the consequence but the cause of infectious diseases. Therefore in his papers up to 1884 we cannot find his definitive opinion on etiological experiments. His final opinion was expressed in the protocol of the 2nd conference about cholera in May 1885 and it was explained in detail in the 1891 publication of his lecture on bacteriological research given to the Tenth International Congress of Medicine in Berlin 1890.

Prof. Dr. Alfred Grafe

Institut für Medizinische Virologie

Im Neuenheimer Feld 324

D-6900 Heidelberg 